ren nur fiir die Operationen empfehlen, wo man eine grössere Menge Cysteninhaltes oder blutigen Serums in der Bauchhöhle zurücklassen muss, resp. den Erguss des letzteren noch erwarten kann, also nach Exstirpation allseitig verwachsener Geschwülste, vorhergegangener Perforation der Cyste in die Bauchhöhle, Morschheit der Wandungen der Geschwulst u. s. w. Die gleichzeitig für Drainage-Fälle von Spiegelberg empfohlene Anwendung der intraperitonealen Behandlung des Stieles, welche auch noch die Reconvalescenz abkürzt, verdient besonders hervorgehoben zu werden. - Genesung nach Versenkung des Stieles und Drainage ist bis jetzt nur ein Mal (Sims) berichtet worden. In dem von mir operirten Falle war die Todestursache besonders in der durch acht Geburten und eine ungenügende Lebensweise sehr verminderten Resistenz des Organismus, 'sowie in der wenig energischen Thätigkeit des fettig infiltrirten Herzens zu suchen. Dresden, 24. Juni 1875.

\title{
Ein Paar Ovariotomien mit versenktem Stiele und Drainage.
}

Von

Otto Spiegelberg.

Die vorstehenden beachtenswerthen Mittheilungen des Herrn Grenser sind mir Veranlassung, einige in der Ueberschrift bezeichnete Erfahrungen bekannt zu geben und einige Bemerkungen daran zu knüpfen; die Wichtigkeit der Drainage und die Spärlichkeit der bezüglichen Casuistik werden dies entschuldigen.

Zuvörderst aber möehte ich bemerken, dass nicht, wie Grenser angiebt, nur ein Mal bislang Genesung nach Versenkung des Stieles und Drainage, und zwar von Sims, berichtet ist, sondern dass dessen 1. und 4. Fall (nach dem Original in New - York med. Journ. 1872/3) hierher gehören. Wie Sims in seinen anderen Fallen mit dem Stiele verfahren, ist nicht recht bestimmt herauszulesen; ich gab früher (dieses Archiv VII, S. 462) an, er habe ihn immer versenkt; es scheint aber bei genaner Durchsicht, dass er den zum Theil künstlich gebildeten auch in der Wunde befestigt hat.

Ich habe drei Mal bei versenktem Stiele drainirt.

1) Rechtsseitige uniloculäre Cyste; Rupțur dersel- 
ben vor Eröffnung; parietale und iliacale Adhäsionen. Genesung.

Frau J. F., Klin. 1873/4, Nr. 38; 46 J., drei Kinder. Alle Functionen in Ordnung. Rechtsseitige Geschwulst seit 5/4 Jahren bemerkt, die in letzter Zeit stark gewachsen und median gerückt ist. Abnahme der Kräfte und Abmagerung.

Befund 16. Juli 1874: Hager. Varicen des rechten Oberschenkels, doppelseitige Cruralhernie. Bauchvenen ektatisch. Sphärische, mehr nach links als rechts gelagerte, frei bewegliche, stark gespannte und fluctuirende Cyste, $20 \mathrm{Cm}$. über die Schamfuge emporragend, Breitenausdehnung in fortlaufender Linie $27 \mathrm{Cm}$; driickt den vorderen Scheidengrund etwas abwärts. Uterus nach rechts hinten gedrängt und unabhängig von der Cyste leicht beweglich.

Die am 17. vorgenommene Probepunction ergiebt bestimmt ovarielle Flïssigkeit.

Ovariotomie am 23. (Drr. Richter - hier, Ananoff Tiflis, Studer - Bern, Stahl - Darmstadt). $6 \mathrm{Cm}$. langer Schnitt, reichliche Menge Peritonealwasser. Eine Deckenarterie an der linken Seite des oberen Wundwinkels muss ligaturirt werden. Das verdickte Netz der vorderen Cystenwand an einzelnen Stellen adhärent. In Folge heftiger Brechbewegungen und starken Gegendruckes von Seiten des Assistenten, um den Darmvorfall zu verhiten, platzt die dünne Sackwand und ihr Inhalt ergiesst sich in die Bauchhöhle. Beim Vorziehen der Cyste müssen mehrere Adhärenzen am Netze, eine breite an der rechten Darmbeingrube, sowie dünne, fadenförmige am Mesenterium des lleum manuell getrennt werden, nachdem die Bauchwunde um $3 \mathrm{Cm}$. nach oben erweitert. Der rechtsseitige Stiel enorm kurz, nur von der hinteren Platte des Ligamentum latum gebildet; die rechte Tuba erweitert, mit dem Stiele durch einige dünne Fäden nur verbunden. Unterbindung des Stieles mit Schonung der Tuba dicht an der Cyste; bei der Abtrennung dieser bleibt ein Stïck derselben am Stiele haften; es wird nachträglich aus seiner serösen Hïlle ausgeschält. - Genaueste Toilette der Bauch - und Beckenhöhle. Im Douglas'schen Raume ein Netz dünner Pseudomembranen, welche ihn rechts besonders förmlich ïberdachen. Drain in gewohnter Weise von oben nach unten durchgeführt (dieses Archiv VII, S. 351). Das lange Netz vor die Därme gelagert. Drei peritoneale und fünf oberflächliche Nähte.

Am ersten Tage floss reichlich blutig gefärbte Flüssigkcit durch die Vagina resp. den Drain $a b$, am zweiten 'Tage etwas weniger, vom dritten an fast gar nichts mehr. Befinden mit Ausnahme starker Kreuzschmerzen und Unmöglichkeit spontanen Harnens sehr gut. Am vierten Tage geringes Durchspilen des Drains mit warmem Wasser, das ziemlich rein grösstentheils aus dem unteren Wundwinkel zurückkommt. Danach heftige Koliken, welche auf Verabreichen einer vollen Opiumdosis sistiren.

Von jetzt an kein Ausfluss aus dem Rohre mehr; doch beibt es noch liegen, da am sechsten Tage die Menstruation eintrat. Eine 
am 7. zum Zwecke klinischer Demonstration ausgefiuhrte Durchspialung des Drains lässt das Wasser rein durch die Scheide abfliessen, doch tritt danach wieder Kolik ein. Am 9. Tage wird der Drain durch die Scheide ausgezogen. Die Nähte wurden successive vom 6. bis 10. 'Tage entfernt; der untere Wundwinkel war in der Tiefe schon 12 Stunden nach Entfernung des Rohres geschlossen. - Im Douglas geringes Exsudat, dessen linke Begrenzung die Punctionsstelle im hinteren Scheidengrunde trifft. - Genesung erfolgte rasch. Am 10. Tage erster spontaner Stuhl, am 8. Tage erste spontane Urinentleerung. Bis zum 15. auch der untere Wundwinkel iuberhäutet. An diesem Tage verlässt die Operirte das Bett und wird am 24. wohl entlassen. Die Exsudation hinter dem Uterus geschwunden, dieser selbst etwas nach rechts gezogen, aber beweglich.

Die Cyste war eine uniloculäre mit papillären Excrescenzen.

2) Rechtsseitiges kystisches Carcinom. Langer Schnitt; feste parietale Adhäsionen Beginnende carcinomatöse Magenerkrankung. Hydropsie. Tod 18 Stunden nach der Operation.

Frau R. H., Klinik 1873/4, Nr. 41. - 54 Jahre, zwei Kinder. Menstruation bis vor einem Jahre regelmässig, seitdem unregelmässige Genitalblutungen. Damit entwickelte sich von der rechten Unterbauchgegend her rapid eine Geschwulst, die schliesslich die Kranke uns zuführte.

Befund Ende Juni 1874: Hochgradige Dyspnoe, vollständige Appetitlosigkeit, kachektisches wachsfarbenes Aussehen, Oedem der ganzen linken Unterextremität und des rechten Unterschenkels. Måssiger Pleuraerguss beiderseits. Urin eiweissfrei. Bauchdecken ödematös in der unteren Hälfte. Sehr breiter massiger, $40 \mathrm{Cm}$. hoch in den Bauch sich erhebender, fester, nicht fluctuirender Tumor von annähernd glatter Oberfläche, seitlich beweglich und ziemlich median gelagert. In den Iliacalgegenden freie Flüssigkeit. Uteruskörper vom Tumor, dessen unteres Segment per vaginam deutlich fühlbar, nach links vorn gedrängt und frei beweglich.

Trotz ungünstiger Prognose, als einziges Rettungsmittel

Ovariotomie am 7. Juli in Anwesenheit einer grosser Zahl fremder Aerzte.

Langer Schnitt bis zu $2 \mathrm{Cm}$. Entfernung vom Nabel; ca. 1 Kilo heller Peritonealflüssigkeit entleert sich. Feste und breite Adhäsionen mit Netz und linksseitiger Parietalserosa, welche letztere theils abgegliaht (Schneideschlinge), theils nach Abbindung mit Messer durchtrennt wurden; an der linken Bauchwand musste ein Stück der äusseren Geschwulsthülle zurückgelassen werden, Der Tumor durch Punction nicht zu verkleinern, erscheint solid, dabei so brichig, dass alle in ihn gesetzten Haken und Zangen sofort ausreissen. Nach Erweiterung der Wunde über den Nabel hinatis auf $24 \mathrm{Cm}$. Totallänge tritt er unter dem Drucke der Hände zu 'T'age. Er entspringt vom hinteren Blatte des rechten Ligamentum latum, die Tube war in ihrer äusseren an der Geschwulst nach oben laufenden Hälfte eng 
mit ihr verbunden. Stiel breit, dünn, aber sehr kurz; wird nach Durchstechung und Unterbindung dicht am Tumor abgetrennt. Wegen starker Blutung an der inneren Seite des Stieles, welche von der Ligatur durchschnitten, neue Unterbindung unterhalb der ersten in drei Portionen. Versenkung; genaueste Entfernung des reichlich in der Beckenhöhle angesammelten Blutes und der Geschwulststückchen. Drainage, 7 peritoneale und 8 oberflächliche Nähte.

Dauer der Operation eine Stunde. - Tiefer Collaps nach derselben. Die Kranke erholt sich aus ihrem Schwächezustande nicht mehr recht und stirbt in demselben 18 Stunden nach der Operation. Durch den Drain war dünne blutig gefärbte Flüssigkeit continuirlich in mässiger Quantität abgeflossen.

Die Section ergab allgemeine Blutleere, doppelseitigen Hydrothorax, geringe fibrinöseitrige Peritonitis, Infiltration der Inguinaldrüsen, Anfangsstadium carcinomatöser Entartung der Magenschleimhaut. Der 5500 Gramm schwere Tumor documentirte sich als ein Kystoma parviloculare papillare carcinomatosum, d. h. als ein Carcinom mit enorm zahlreichen kleinen, zum Theil von papillaren Epithelwucherungen, zum Theil von Extravasaten erfüllten Hohlräumen.

3) Rechtsseitiges multiloculäres Cystom. Diffuse parietale Adhäsionen. Dünne brüchige Cystenwand. Ge nesung.

Frau F. (privat), 52 Jahre; hat ein Kind geboren, seit drei Jahren Menopause. Anschwellung des Leibes schon vor drei Jahren bemerkt; gleichzeitig entwickelte sich Procidenz der Scheide und der Gebärmutter. Vor ungefähr zwei Jahren an „Blinddarmentzuindung" behandelt.

Befund am 7. Mai 1875: Kräftige hagere Person, klagt nur über mechanische Beschwerden im Leibe. Rechte Seite desselben druckempfindlich. Prominenter cystöser Tumor von der Grösse des hochschwangeren Uterus, $38 \mathrm{Cm}$. hoch; sphärisch, prall, deutlich multiloculär; median, beweglich: Uterus retroflectirt, Collum sehr lang und atrophisch; vordere Vaginalwand prolabirt; im Beckeneingange vor dem Uterus der Tumor voll zu fühlen und Fluctuation von aussen hier zu erzeugen. Sondenerhebung des Uterus unmöglich, Versuch dazu sehr schmerzhaft; über dem rechten Vaginalgrunde das strangähnlich verdickte Ligamentum latum zu fühlen.

Probepunction am 19. ergiebt helle schleimige dickliche Flüs: sigkeit ovariellen Aussehens, aber auch mit spontaner Gerinnselbildung nach einiger Zeit.

Ovariotomie am 22. [Drr. Richter, Fuhrmann, Landau, Rubinstein - Warschau, Jacubovits - Moskan, Hempel]. $8 \mathrm{Cm}$. langer Schnitt, eine Ligatur im oberen Wundwinkel; dicke Serosa. Die dünne vorliegende Cystenwand wird bei Durchschneidung des Peritoneums eröffnet, die Oeffnung aber durch Zangen bald geschlossen. Meso- und epigastrische, besonders rechts ausgebreitete Adhäsionen werden leicht getrennt. Beim Vorziehen der Geschwulst reisst ihre ausserordentlich dünne und brüchige Wand 
wiederholt ein, und es fliesst viel schleinige gleichmässig gemischte Flïssigkeit in die Bauchhöhle. Im hinteren Bauch- und Beckenraume lagern umfangreiche Cystenconglomerate, die von dem Hauptsacke aus entleert werden; dieser aber bricht beim Vorziehen immer wieder durch. Schliesslich wird die Gesammtmasse durch Umgreifen an ihrer Rïckseite zu 'Tage gebracht, ohne dass es nöthig war, die Bauchwunde weiter zu verlängern.

Kurzer und dünner rechtsseitiger Stiel, der nur aus dem Ligamentum ovarii und dem Mesosalpinx bestand; Tube selbst frei. Klammer, und Abtrennung der Cyste an der Insertionsstelle des Stieles in dieselbe. Dabei kommt bei der Kürze des Stieles die rechte Uteruskante in die Bauchwunde, und es will der brüchige dünne stiel aus der Klammer zurïckschlüpfen. Er wird deshalb unter letzterer durchstochen und unterbunden, nachdem eine gewisse Quantität Blut aus ihm in die Beckenhöhle geflossen. Der Uterus ist in seinem supravaginalen Halstheile sehr verlängert und atrophisch; seine linken Anhänge sind gesund, wie sie es auch in den ersten beiden Fällen waren. - Sorgfältige Reinigung der Bauch - und Beckenhöhle. Der Douglas'sche Raum ist sehr weit nach unten verlängert, seine Wände faltig, er ist ganz von Darmschlingen erfüllt. An dem tiefsten Punkte seiner vorderen Wand wird der Drain durchgeführt und nach nochmaliger Reinigung der Beckenhöhle, Besichtigung des Stieles, Abschneiden der Ligaturfäden dicht am Knoten, die Bauchwunde durch fünf einander wahe peritoneale und einige oberflächliche Nähte geschlossen. - Operationsdauer 40 Minuten.

Die Reaction am ersten und zweiten Tage war ziemlich stark, bei hohem Fieber aber schwitzte Patientin enorm. Durch den Drain flossen grosse Mengen blutiger dünner Flüssigkeit fortwährend ab. Dies liess am 3. Tage nach und wurde an diesem auch zuerst der Verband erneuert; der Bauch war jetzt ein wenig im Epigastrium aufgetrieben, die Wunde vom besten Aussehen. Spontaner Stuhl am selbigen Tage. - Von jetzt ab stetige Erholung; vom 5. 'Tage an waren die Unterlagen ganz trocken; Exsudat im Douglas nie zu fühlen. Doch liess ich den Drain immer noch liegen und entfernte ihn bei dem guten Zustande der Genesenden erst am 8. 'Tage. Die Nähte wtrden vom 4.-7. Tage successive entfernt; an der Stelle der abersten peritonealen Metallnaht, die beim ersten neuen Verbande abgebrochen und deren Enden im Fleische retrahirt waren, hatte sich ein kleiner Abscess gebildet. Die dem Drain entsprechende Stelle im unteren Wundwinkel war schon am Abend nach seiner Entfernung in der Tiefe geschlossen und ging 'schnell der Ueberhäutung entgegen. Am 13. Tage verliess bei gutem Befinden die Patientin ibr Bett und reiste am 23. nach Hause. Wunde vernarbt; Uterus gerade, in mittlerer Stellung, beweglich; Douglas frei.

Die Geschwnlst erwies sich als ein Cystoma multiloculare glandulare. Die Haupteyste war fast iiberall ilhres Epithels beraubt and zeigte auf ihrer Innenfläche eine rabenkieldicke lange thrombosirte Vene mit vielen feinen ebenfalls thrombosirten Aesten. Ihr Zellinhalt glich 
Wanderzellen, und es lässt sich daraus vielleicht erklären, dașs in der Punctionsflüssigkeit etwas spontan gerinnende Substanz gefunden war. (Dr. Weigert.)

In Bezug auf Werthschätzung der Drainage kann ich wohl vom zweiten Falle, in welchem die Operation ja nur als letzter Rettungsversuch ausgeführt wurde und der lethale Ausgang durch die Schwere der Allgemeinerkrankung bedingt war, absehen. Der milde Verlauf der Genesung in den beiden anderen Fällen aber ist evident. Und ich muss ihn wesentlich der Drainage zuschreiben nach den Erfahrungen, welche ich sonst bei reichlichem Ergusse von Cysteninhalt und Blut in die Bauchhöhle und bei versenktem Stiele gemacht habe. In letzter Beziehung habe ich fast jedes Mal eine mehr weniger massenhafte Exsudation um den Stiel beobachtet, welche in zwei Fällen sogar später noch zum Durchbruche durch die.äussere Wunde resp. zu secundärer Peritonitis führte, während in den beiden obigen Beobachtungen jene in erster höchst gering war und in der anderen ganz fellte. Und was soll aus der serösblutigen Masse in der Beckenhöhle werden, wie sie in beiden Fällen ausfloss, wenn dies letztere nicht eben durch den Drain ermöglicht ist? Wenn also gegen die Berechtigung der Drainage in derartigen Fällen a priori gar kein Einwand erhoben werden konnte, so ist es doch gewiss nicht ohne Nutzen, dieselbe auch durch die thatsächliche Beobachtung zu beweisen und die vielleicht noch hier und da bestehenden Gegner damit zu ihr zu bekehren.

Der wesentlichste Nutzen der sofortigen Drainage scheint mir indess darin zu liegen, dass sie mit Wegschaffen der Ansammlung von, in der Operation ergossener und frisch nach transsudirter Fliussigkeit den Eintritt des Zerfalles derselben auch unmöglich macht. Ist es jedoch zur Eiter- resp. Jauchebildung gekommen, so ist der Werth der Drainage sehr problematisch; wenn auch immer nützlich und bisweilen rettend, so ist sie doch dann in den meisten Fällen nicht genügend, weil die Säfteinfection schon gesetzt ist und die eitrig. jauchigen Ansammlungen so häufig an Stellen sich befinden, zu welchen der Drain eben keinen Zugang hat oder von denen vielmehr kein Zugang zum Drain zu ermöglichen ist. Weil es sich also wesentlich um Verhütung jauchigen Zerfalles handelt, halte ich die Durchspülung des Drains für durchaus verboten, so lange kein Zeichen septischer Processe resp. Resorption vorliegt. Denn wird jene Procedur auch mit den besten Cautelen ausgeführt, fremde, Zersetzung erregende Stoffe können doch gar leicht mit eindringen; und schon der mechanische Insult der, Füllung der Beckenhöhle ist für das Bauchfell und die in jener enthaltenen Organe nicht gleichgiltig. Fliesst bei sonst gutem Wohlbefinden nichts aus dem Drain ab, so ist das ja sehr gut; glaubt man eine Verstopfung desselben annehmen zu müssen, so dislocire man ihn, wie ich schon früher (VII, S. 352) bemerkt, so, dass man den in der Bauchhöhle liegendęn Theil heraus 
und den bis dahin aussen befindlichen, nachdem man ihn wohl geprifft und gereinigt, hinein zieht. Deshalb soll das Rohr auch wenigstens 1 Mr. lang sein. Eine Einklemmung habe ich nie gesehen, weil ich, auch in anderen Fällen, nie genöthigt war, den Drain länger als acht Tage liegen zu lassen; in dieser Zeit ist sie aber auch bei ganz dünner Röhre. nicht zu fürchten, eher später, wenn es zur Narbencontraction in der Umgebung der Punctionsstelle kommt. Ich halte es jedoch immer für das Sicherste, so früh wie möglich das Drainrohr zu entfernen, also sobald nichts als höchstens eine ganz kleine Quantität guten Eiters in ihm sich befindet und der sonstige Zustand gut ist; man bedarf des Drains dann auch nicht mehr als eines etwaigen Boten von Blutung, da bei gut angelegten Ligaturen diese, wenn sie nicht alsbald nach der Operation erfolgt, nicht mehr zu erwarten ist.

Sehr beachtenswerth ist, was Grenser über die Bildung eines Kanales zwischen Scheide und Mastdarm beim Durchstechen von der Bauchhöhle aus sagt ${ }^{1}$ ). Aber dieses Ereigniss ist sicherlich zu vermeiden, wenn man bimanuell verfährt, wie ich es auf der hiesigen Naturforscher-Versammlung sagte. Der Operateur muss die Canüle des genügend langen und dicken gebogenen Trokars mit zurückgezogenem Stilet an der tiefsten Stelle der vorderen Wand des Douglas'schen Raumes fixiren, und zwar muss er dies selbst thun, damit nicht andere als seine Hände in die Bauchhöhle gelangen, und weil er ja am besten in der Operation schon über die individuellen Verhältnisse der Beckenhöhle orientirt ist; die Gefahr, eine der im Douglas liegenden Darmschlingen zu treffen, ist, wie ich selbst erfahren, nicht gering. Den Punkt, an welchem die Canïle den hinteren Scheidengrund etwas vorstülpt, umstellt der Assistent mit Zeige- und Mittelfinger genau, so dass die 'Trokarspitze zwischen ihnen durchtreten muss. Ist so die Perforationsstelle genau gesichert, so fuihrt der Assistirende, um seine Finger zu schonen, selbst das Stilet mit raschem Stosse zwischen ihnen durch, während der Operateur immer noch die Canïle genau an der ursprünglichen Stelle fest angedrückt hält. Und erst wenn der Assistent die Spitze des Instrumentes frei in der Scheide fühlt, übergiebt man ihm auch die Canüle und lässt ihn den ganzen Trokar durchstossen. So perforirt man den Douglas auf dem kürzesten. Wege, und zur Bildung eines falschen Weges kann es absolut nicht kommen.

1) Uebrigens hat Sims nicht in seinem 5. Falle von oben nach unten durchgestochen, sondern schon im 1. und 4. (des Originals). 